Helsinki University of Technology Laboratory for Theoretical Computer Science Technical Reports 23

Teknillisen korkeakoulun tietojenkäsittelyteorian laboratorion tekninen raportti 23

\title{
SYMBOLIC MODEL CHECKING OF HIERARCHICAL UML STATE MACHINES
}

Jori Dubrovin and Tommi Junttila 

Helsinki University of Technology Laboratory for Theoretical Computer Science Technical Reports 23

Teknillisen korkeakoulun tietojenkäsittelyteorian laboratorion tekninen raportti 23

\section{SYMBOLIC MODEL CHECKING OF HIERARCHICAL UML STATE MACHINES}

Jori Dubrovin and Tommi Junttila

Helsinki University of Technology

Department of Computer Science and Engineering

Laboratory for Theoretical Computer Science

Teknillinen korkeakoulu

Tietotekniikan osasto

Tietojenkäsittelyteorian laboratorio 


\section{Distribution:}

Helsinki University of Technology

Laboratory for Theoretical Computer Science

P.O.Box 5400

FI-02015 TKK, FINLAND

Tel. +35894511

Fax. +35894513369

E-mail: lab@tcs.tkk.fi

URL: http://www.tcs.tkk.fi/

(C) Jori Dubrovin and Tommi Junttila

ISBN 978-951-22-9191-5

ISSN 0783-540X

Multiprint 0y

Espoo 2007 
ABSTRACT: A compact symbolic encoding is described for the transition relation of systems modeled with asynchronously executing, hierarchical UML state machines that communicate through message passing and attribute access. This enables the analysis of such systems by symbolic model checking techniques, such as BDD-based model checking and SAT-based bounded model checking. Message reception, completion events, and run-to-completion steps are handled in accordance with the UML specification. The size of the encoding for state machine control logic is linear in the size of the state machine even in the presence of composite states, orthogonal regions, and message deferring. The encoding is implemented for the NuSMV model checker, and preliminary experimental results are presented.

KEYWORDS: UML semantics, UML state machine, symbolic model checking, verification 



\section{CONTENTS}

1 Introduction $\quad 1$

1.1 Related Work . . . . . . . . . . . . . . . . . . . . . 2

2 A UML Subset and its Semantics 2

2.1 Types, Signals, and Classes . . . . . . . . . . . . 3

2.2 State Machines . . . . . . . . . . . . . . . . 3

2.3 Systems and State Spaces . . . . . . . . . . . . . . . . 6

3 Encoding System Behavior 9

3.1 State Variables . . . . . . . . . . . . . . . . . . . . . . 99

3.2 Queues and Messages . . . . . . . . . . . . . . . . . 10

3.3 Control Logic of State Machines . . . . . . . . . . . . . . . 11

3.4 Analysis . . . . . . . . . . . . . . . . 15

3.5 Preliminary Evaluation . . . . . . . . . . . . 16

4 Conclusions 17

$\begin{array}{ll}\text { References } & 17\end{array}$ 



\section{INTRODUCTION}

Model checking [7] is an automatic way of verifying that a hardware or software system fulfills its behavioral requirements. In symbolic model checking, the behavior of a system is analyzed by manipulating sets of states instead of individual states, often leading to remarkable speedup in verification time. A prerequisite for applying such techniques is a symbolic encoding for the transition relation of the system.

The main contribution of this paper is a compact symbolic encoding for the control logic of communicating UML state machines [22]. This directly enables one to use state-of-the-art symbolic model checking techniques such as BDD-based [17] and bounded model checking [4] to UML systems composed of asynchronously executing, message passing state machines. The encoding is implemented in a tool that translates UML models to the input language of the symbolic model checker NuSMV [6]. In the perspective of Model Driven Engineering (MDE), the presented encoding is in principle sufficient for analyzing system models in early design phases when they do not yet contain too many data structures but only a communication skeleton. Analyzing models in later design phases requires in practice the use of model reduction techniques such as slicing and abstraction in addition.

The second contribution is that we give an accurate semantics for the subset of UML models we consider. The semantics is well suited for symbolic model checking because it (i) fixes the atomicity level, (ii) does not contain any pseudo-code but works directly on the state space level, and (iii) handles asynchronously executing objects, classes, and data (including signal parameters often omitted in similar works) in an abstract yet exact level without fixing the actual action description language or the type system. Special care is taken to formalize run-to-completion steps in accordance with [22].

The main criterion for the selected UML subset is that it is suitable for describing systems composed of asynchronously executing objects communicating with message passing, such as communication protocols. Therefore, asynchronous signal events are included in the subset but synchronous call events are not. Of UML state machine features we support (i) state hierarchy, which is important for compactness and clarity of models, (ii) deferring of messages, (iii) completion events, (iv) concurrent composite states, and (v) initial and choice pseudostates. The only restriction is that we do not allow concurrent substates to react to the same signal because we feel that it is an inferior way of intra-object synchronization. As a result, the semantics becomes easier to understand and to use as a basis for model checking and code generation.

One way to deal with hierarchical state machines is to flatten the hierarchy away as a preprocessing step. However, in the presence of concurrent composite states, this can result in an exponential blowup in the number of states of the flattened state machine. If there are no concurrent composite states (or history pseudostates), then flattening will not add new states but the number of transitions can become quadratic in the size of the original state machine. The encoding presented here is not based on flattening but instead builds on the hierarchical structure of states. As a result, the control logic encoding can be represented in linear size w.r.t. the original state machine. 


\subsection{Related Work}

A lot of research has already been done both on the semantics and on symbolic encodings of UML state machines, see e.g. [3,8] for surveys. In the following we compare our work to the most relevant works in these areas.

The semantics presented in this paper is most closely related to that in [16]. The main difference is that while [16] presents a pseudo-code algorithm that executes UML models, the semantics in this paper is presented as a relation between successive configurations of the system, making it explicit what a single step of execution is. Such a transition relation is required for applying symbolic model checking.

A semantics for hierarchical UML state machines is presented in [14], but signal parameters and data manipulation are not discussed. In [23], the semantics is refined and a superlinear size symbolic encoding is presented. However, the processing of event queues is not thoroughly explained.

As a part of a larger EU project, [9] presents a symbolic transition relation for UML state machines (including call events which are not considered in this paper) but does not handle hierarchy or deferring of events.

In [2], a semantics for UML state machines and an approach for applying an interactive theorem prover is given. Choice pseudostates, signal parameters, and deferring of events are not supported, and the interaction between concurrently executing state machines is not discussed. A translation from UML state machines to NuSMV programs is sketched in [20], but completion events are not supported and signal parameters, deferring of events, handling of transition priorities, concurrent composite states, or the exact semantics of state machines are not discussed in detail.

The semantics in [13] is based on translating the full UML 2.0 state machine language to (superlinear size) "core state machines". The granularity of the execution semantics is much finer than in this work, and we do not know of symbolic encodings based on [13].

Furthermore, none of $[13,9,14,20,23]$ seem to handle completion events in full accordance with [22]; see Sect. 2.2 on "quiescent states" below.

\section{A UML SUBSET AND ITS SEMANTICS}

This section defines the class of UML systems considered in this paper. In a nutshell, a UML system is composed of a finite set of objects that are instances of classes in the underlying UML model. The objects can communicate with each other via asynchronous message passing and by accessing each other's attributes. The behavior of each object is described by the hierarchical state machine associated with the class of the object.

We feel that a detailed formal definition such as the one below is justified and valuable in order to ensure correct handling of non-trivial features of UML state machines including hierarchy, deferring and implicit consumption of messages, message parameter reception, completion events, and quiescing. 


\subsection{Types, Signals, and Classes}

As the focus of this report is on UML state machines, other relevant parts of UML models, e.g. data attribute manipulation, are defined only in a very abstract way.

To capture data types in UML models, a finite set $\mathcal{T}$ of types is assumed, each type $T \in \mathcal{T}$ being associated with a non-empty domain set $\operatorname{dom}(T)$. In particular, the Boolean type $\mathbb{B}$ with $\operatorname{dom}(\mathbb{B})=\{$ false, true $\}$ belongs to $\mathcal{T}$. A typed variable is a name $x$ associated with a type type $(x) \in \mathcal{T}$. The guards and effects appearing in state machines are expressed with a strongly typed action language $\mathcal{L}$ over the types; $\mathcal{L}_{\mathbb{B}} \subset \mathcal{L}$ denotes the set of side-effect free Boolean valued expressions and $\mathcal{L}_{\text {Stmt }} \subset \mathcal{L}$ the set of (possibly compound) statements. One example of such an action language is given in [10].

As usual, the set of all finite sequences over a set $X$ is denoted by $X^{*}$. If $a=\left\langle a_{1}, \ldots, a_{k}\right\rangle \in X^{*}, b=\left\langle b_{1}, \ldots, b_{l}\right\rangle \in X^{*}$, and $x \in X$, then append $(a, x)=\left\langle a_{1}, \ldots, a_{k}, x\right\rangle$, dequeue $(a)=\left\langle a_{2}, \ldots, a_{k}\right\rangle$ (undefined if $k=0)$, and concat $(a, b)=\left\langle a_{1}, \ldots, a_{k}, b_{1}, \ldots, b_{l}\right\rangle$.

Objects can communicate with each other by sending messages built over a finite set Sigs of signals. Each signal sig $\in$ Sigs is associated with a list params $($ sig $)=\left\langle T_{\text {sig, } 1}, \ldots, T_{\text {sig, } k_{s i g}}\right\rangle \in \mathcal{T}^{*}$ of parameter types. A message is of the form $\operatorname{sig}\left[v_{1}, \ldots, v_{k_{s i g}}\right]$, where $\operatorname{sig} \in \operatorname{Sigs}$ and each $v_{i} \in \operatorname{dom}\left(T_{\text {sig }, i}\right)$; the set of all messages is denoted by Msgs. Message reception in state machines is denoted by signal triggers of the form $\operatorname{sig}\left(x_{1}, \ldots, x_{k_{s i g}}\right)$, where $\operatorname{sig} \in$ Sigs and each $x_{i}$ is a typed variable with type $\left(x_{i}\right)=T_{\text {sig, } i}$. The set of all signal triggers is denoted by Trigs.

A class is a pair $C=\langle\operatorname{attrs}, s m\rangle$, where $\operatorname{Attrs}(C)=\operatorname{attrs}$ is a finite set of typed variables called attributes and $S M(C)=s m$ is the state machine of the class.

\subsection{State Machines}

The behavior of an instance of a class (i.e. an object) is described by the associated state machine. Formally, a hierarchical UML state machine is a structure

$$
\text { sm }=\langle\mathcal{S}, \mathcal{R}, \text { top, container, } \mathcal{T} \text {, defers }\rangle,
$$

where

- $\mathcal{S}$ is a finite set of state vertices partitioned into simple states $\mathcal{S}_{s i}, \mathrm{com}$ posite states $\mathcal{S}_{c o}$, final states $\mathcal{S}_{f i}$, initial pseudostates $\mathcal{S}_{i n}$, and choice pseudostates $\mathcal{S}_{c h}$

- $\mathcal{R}$ is a finite set of regions (disjoint from $\mathcal{S}$ );

- top $\in \mathcal{R}$ is the unique top region;

- container $:(\mathcal{S} \cup \mathcal{R} \backslash\{$ top $\}) \rightarrow(\mathcal{S} \cup \mathcal{R})$ describes the state hierarchy of the state machine;

- $\mathcal{T}$ is a finite set of transitions; and 


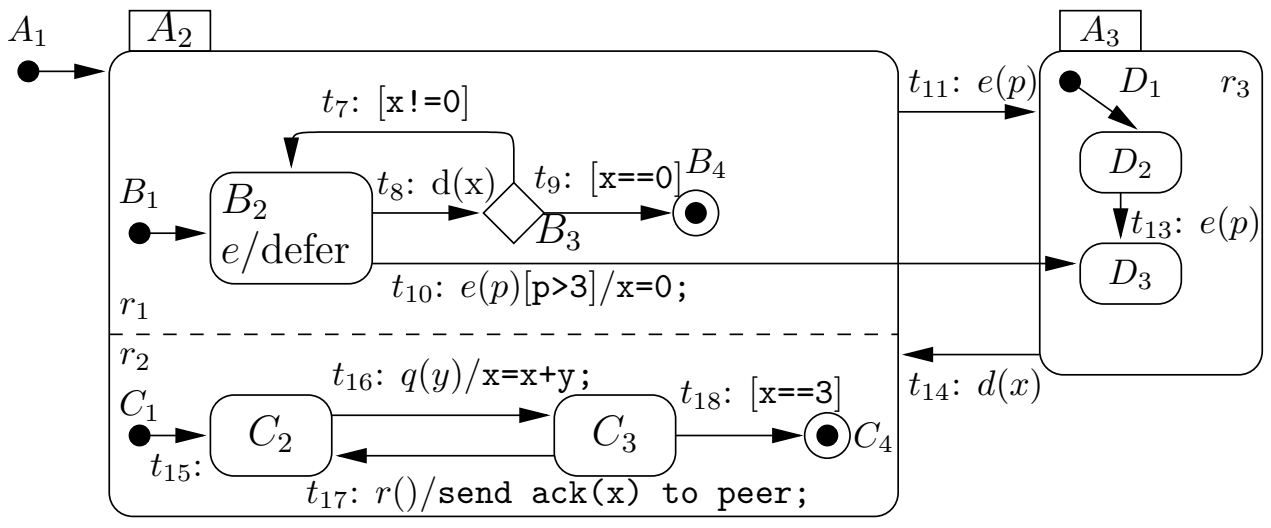

Figure 1: A UML state machine.

- defers : $\left(\mathcal{S}_{s i} \cup \mathcal{S}_{c o}\right) \rightarrow 2^{\text {Sigs }}$ assigns each state a (possibly empty) set of deferrable signals.

For each state vertex or region $v \in \mathcal{S} \cup \mathcal{R}$ of the state machine, define the functions children $(v)=\left\{v^{\prime} \in \mathcal{S} \cup \mathcal{R} \backslash\{\right.$ top $\left.\} \mid \operatorname{container}\left(v^{\prime}\right)=v\right\}$ and descendants $(v)=\left\{v^{\prime} \in \mathcal{S} \cup \mathcal{R} \backslash\{\right.$ top $\} \mid \exists i>0$ : $\left.\operatorname{container}^{i}\left(v^{\prime}\right)=v\right\}$. It is required that the container of each non-top region is a composite state, and that the container of each state vertex is a region. The state hierarchy must be a connected tree, i.e. descendants $($ top $)=\mathcal{S} \cup \mathcal{R} \backslash\{$ top $\}$ must hold. Furthermore, each region must contain exactly one initial state, i.e. $\forall r \in$ $\mathcal{R}:\left|\operatorname{children}(r) \cap \mathcal{S}_{i n}\right|=1$, and each composite state at least one region, i.e. $\forall s \in \mathcal{S}_{c o}: \operatorname{children}(s) \neq \emptyset$. If a composite state contains more than one region, then it is called concurrent. Two state vertices $s_{1}, s_{2} \in \mathcal{S}$ are orthogonal, denoted $s_{1} \perp s_{2}$, if there are distinct regions $r_{1}, r_{2} \in \mathcal{R}$, $r_{1} \neq r_{2}$ such that container $\left(r_{1}\right)=\operatorname{container}\left(r_{2}\right), s_{1} \in \operatorname{descendants}\left(r_{1}\right)$, and $s_{2} \in$ descendants $\left(r_{2}\right)$. A set $S \subseteq \mathcal{S}$ of states is consistent iff for any two distinct state vertices $s_{1}, s_{2} \in S$ either $s_{1} \perp s_{2}, s_{1} \in \operatorname{descendants}\left(s_{2}\right)$, or $s_{2} \in \operatorname{descendants}\left(s_{1}\right)$.

As an example, consider the state machine in Fig. 1. $A_{2}$ is a concurrent composite state with container $\left(A_{2}\right)=$ top and children $\left(A_{2}\right)=\left\{r_{1}, r_{2}\right\}$, where $r_{1}$ and $r_{2}$ are regions. $B_{2}$ is a simple state with $\operatorname{defers}\left(B_{2}\right)=\{e\}$ and container $\left(B_{2}\right)=r_{1}$. The choice pseudostate $B_{3}$ and the final state $C_{4}$ are orthogonal. The state set $\left\{A_{2}, B_{1}, C_{2}\right\}$ is consistent while $\left\{A_{3}, D_{2}, D_{3}\right\}$ is not.

A transition $t$ in the set $\mathcal{T}$ of transitions is a tuple

$$
\left\langle s, \sigma, g, e, s^{\prime}\right\rangle \in\left(\mathcal{S} \backslash \mathcal{S}_{f_{i}}\right) \times(\text { Trigs } \cup\{\tau\}) \times \mathcal{L}_{\mathbb{B}} \times \mathcal{L}_{\mathrm{Stmt}} \times\left(\mathcal{S} \backslash \mathcal{S}_{i n}\right) .
$$

We define source $(t)=s$, guard $(t)=g$, effect $(t)=e$, and target $(t)=s^{\prime}$. The container container $(t)$ of $t$ is the smallest (w.r.t. the partial order induced by container) region such that $\left\{s, s^{\prime}\right\} \subseteq$ descendants $(r)$. If $\sigma=\tau$, we say that $t$ is a completion transition and define triggersig $(t)=\tau$. Otherwise, $\sigma=\operatorname{sig}(\ldots)$ and we define triggersig $(t)=\operatorname{sig}$. In UML only completion transitions can leave pseudostates: source $(t) \in \mathcal{S}_{\text {in }} \cup \mathcal{S}_{\text {ch }}$ implies triggersig $(t)=\tau$. We require that transitions originating from orthogonal states are not triggered by the same signal: for all $t_{1}, t_{2} \in \mathcal{T}$, if 
triggersig $\left(t_{1}\right)=\operatorname{triggersig}\left(t_{2}\right) \neq \tau$, then $\operatorname{source}\left(t_{1}\right) \perp$ source $\left(t_{2}\right)$ must not hold.

In Fig. 1, transition $t_{10}=\left\langle B_{2}, e(p), \mathrm{p}>3, \mathrm{x}=0 ;, D_{3}\right\rangle$ has container $\left(t_{10}\right)=$ top. The completion transition $t_{15}$ has container $\left(t_{15}\right)=r_{2}, \operatorname{guard}\left(t_{15}\right)$ is implicitly true, and effect $\left(t_{15}\right)=$ skip, where skip is a pseudostatement that does nothing.

State Configurations. A state configuration of the state machine is a pair

$$
s c=\langle A, Q\rangle
$$

where the set $A$ of active state vertices is a maximal consistent subset of $\mathcal{S}$ and $Q \subseteq A$ is a set of quiescent states. The intuition is that a state is in $Q$ if it has already consumed its implicit completion event. A completion event of a state is consumed either by firing an outgoing completion transition or, if the guards of all completion transitions evaluate to false, by quiescing the state, after which the completion transitions will not become enabled even if the guards become true. This construction (from [16]) accurately models the requirement $[22$, p. 659] that the guards of completion transitions will not be evaluated again without re-entering the state. For example, state $C_{3}$ in Fig. 1 can become quiescent if $\mathrm{x}==3$ does not hold, and then $t_{18}$ will not become enabled even if the value of $\mathrm{x}$ is changed to 3 .

Because completion events are only relevant for states with outgoing completion transitions, we only define quiescence status for the set of completion sensitive states $\mathcal{S}_{C S}=\left\{s \in \mathcal{S}_{s i} \cup \mathcal{S}_{c o} \mid \exists t \in \mathcal{T}\right.$ s.t. source $(t)=$ $s$ and triggersig $(t)=\tau$ \}. Thus, $Q$ is always a subset of $A \cap \mathcal{S}_{C S}$. We say that a state $s \in \mathcal{S}_{C S}$ is ready to consume its completion event in $s c$, denoted by ceready $(s c, s)$, if

1. it is active but not quiescent: $s \in A \backslash Q$, and

2. it is either (i) a simple state: $s \in \mathcal{S}_{s i}$, or (ii) a composite state with all its regions in final states: $s \in \mathcal{S}_{c o}$ and $\forall s^{\prime} \in A: \operatorname{container}^{2}\left(s^{\prime}\right)=s \Rightarrow$ $s^{\prime} \in \mathcal{S}_{f i}$.

A state configuration $s c=\langle A, Q\rangle$ is

- in compound transition, denoted by inct $(s c)$, if it contains an active pseudostate: $A \cap\left(\mathcal{S}_{i n} \cup \mathcal{S}_{c h}\right) \neq \emptyset$,

- in run-to-completion (RTC) step, denoted by $\operatorname{inrtc}(s c)$, if (i) it is in compound transition, or (ii) there is a completion sensitive state that is ready to consume its completion event in it, and

- stable, denoted by stable (sc), if it is not in RTC step.

A UML state machine consumes messages from its input queue only when it is in a stable state configuration.

Consider again the state machine in Fig. 1. The state $C_{3}$ is the only completion sensitive state in it. The pair $\left\langle\left\{A_{2}, B_{2}, C_{3}\right\},\left\{C_{3}\right\}\right\rangle$ is a stable state configuration, while the state configuration $\left\langle\left\{A_{2}, B_{3}, C_{3}\right\},\left\{C_{3}\right\}\right\rangle$ is in compound transition. The state configuration $\left\langle\left\{A_{2}, B_{2}, C_{3}\right\}, \emptyset\right\rangle$ is in RTC step 
(but not in compound transition) because the state $C_{3}$ is ready to consume its completion event in it.

The default entry completion of a state vertex $s \in \mathcal{S}$, denoted by $\operatorname{dec}(s)$, is the smallest maximal consistent subset of $\mathcal{S}_{c o} \cup \mathcal{S}_{i n} \cup\{s\}$ such that $s \in \operatorname{dec}(s)$. In the state machine in Fig. $1, \operatorname{dec}\left(A_{3}\right)=\left\{A_{3}, D_{1}\right\}, \operatorname{dec}\left(D_{3}\right)=\left\{A_{3}, D_{3}\right\}$, $\operatorname{dec}\left(C_{3}\right)=\left\{A_{2}, B_{1}, C_{3}\right\}$, and $\operatorname{dec}\left(A_{2}\right)=\left\{A_{2}, B_{1}, C_{1}\right\}$.

Given a state configuration $s c=\langle A, Q\rangle$ and a transition $t \in \mathcal{T}$ with source $(t) \in A$, the $t$-successor of $s c$ is

$$
\operatorname{succ-conf}(s c, t)=\left\langle A^{\prime}, Q^{\prime}\right\rangle,
$$

where $A^{\prime}=(A \backslash D) \cup(\operatorname{dec}(\operatorname{target}(t)) \cap D), Q^{\prime}=Q \backslash D$, and $D$ is a shorthand for descendants (container $(t))$. In the state machine in Fig. 1, the $t_{10}$-successor of the state configuration $s c=\left\langle\left\{A_{2}, B_{2}, C_{3}\right\},\left\{C_{3}\right\}\right\rangle$ is $\left\langle\left\{A_{3}, D_{3}\right\}, \emptyset\right\rangle$ while the $t_{11}$-successor of $s c$ is $\left\langle\left\{A_{3}, D_{1}\right\}, \emptyset\right\rangle$.

\subsection{Systems and State Spaces}

We consider a UML system to consist of a finite set of objects $O$, each object $o \in O$ being associated with the class Class(o) that it is an instance of. A global configuration of the system is a tuple

$$
g c=\left\langle\text { stateconf }_{g c}, \text { attrvals }_{g c}, \text { inputq }_{g c}, \text { deferq }_{g c}\right\rangle,
$$

where

- stateconf $_{g c}$ maps each object $o$ to the current state configuration of its state machine $S M(\operatorname{Class}(o))$,

- attrvals $_{g c}$ maps each object $o$ to a function giving each attribute $x \in$ $\operatorname{Attrs}(\operatorname{Class}(o))$ its current value in dom $($ type $(x))$, and

- inputq $_{g c}$ deferq $_{g c}: O \rightarrow$ Msgs $^{*}$ describe the contents of the input and deferred queues, respectively, of each object.

For convenience, let StateConf $(g c, o)=\operatorname{stateconf}_{g c}(o), \operatorname{AttrVal}(g c, o, x)=$ attrvals $_{g c}(o)(x), \operatorname{Input} Q(g c, o)=$ inputq $_{g c}(o)$, and let DeferQ $(g c, o)=$ deferq $_{g c}(o)$. The set of all global configurations is denoted by $\mathcal{G C}$.

Given a side-effect free Boolean expression $\phi$ in $\mathcal{L}_{\mathbb{B}}$, eval $(g c, o, \phi)$ evaluates it in the context of a global configuration $g c$ and an object $o$, and returns false or true. Given a statement $\gamma$ in $\mathcal{L}_{\text {Stmt }}$, $\operatorname{exec}(g c, o, \gamma)$ executes it in the context of $g c$ and $o$, and returns a new global configuration $g c^{\prime}$ with the restrictions that, for each $o^{\prime} \in O$, (i) the state configuration is not modified: StateConf $\left(g c^{\prime}, o^{\prime}\right)=$ StateConf $\left(g c, o^{\prime}\right)$, (ii) messages cannot be removed from the input queue: $\operatorname{Input} Q\left(g c, o^{\prime}\right)$ is a prefix of $\operatorname{Input} Q\left(g c^{\prime}, o^{\prime}\right)$, and (iii) the deferred queue is not modified: $\operatorname{Defer} Q\left(g c^{\prime}, o^{\prime}\right)=\operatorname{Defer} Q\left(g c, o^{\prime}\right)$.

UML requires that for each pseudostate, there is always at least one outgoing transition whose guard is true: $\forall g c \in \mathcal{G C}, \forall o \in O, \forall s \in \mathcal{S}_{i n} \cup \mathcal{S}_{c h}: \exists t \in \mathcal{T}$ s.t. $\operatorname{source}(t)=s$ and $\operatorname{eval}(g c, o, \operatorname{guard}(t))=$ true. 
State Spaces. The actual semantics of a UML system is given by its state space that describes how the system may evolve from one global configuration to another. Each atomic step between global configurations corresponds to one object either firing one transition, deferring a message, or implicitly consuming a message or a completion event. The UML run-to-completion semantics for individual state machines is followed as messages can only be consumed in stable state configurations. Formally, the state space of a UML system is the tuple

$$
\left\langle\mathcal{G C}, g c_{\text {init }}, \Delta\right\rangle,
$$

where $g c_{\text {init }} \in \mathcal{G C}$ is the initial configuration, and $\Delta \subseteq \mathcal{G C} \times \mathcal{A} \times \mathcal{G C}$ is the minimal transition relation defined by the following rules $(\mathcal{A}$ being a set of possible annotations). Assume an object $o \in O$, that $S M(\operatorname{Class}(o))=$ $\langle\mathcal{S}, \mathcal{R}$, top , container, $\mathcal{T}$, defers $\rangle$ and let $s c=\langle A, Q\rangle=\operatorname{State} \operatorname{Conf}(g c, o)$.

- Signal Triggered Transitions. Let $t=\left\langle s, \operatorname{sig}\left(x_{1}, \ldots, x_{k}\right), g, e, s^{\prime}\right\rangle \in$ $\mathcal{T}$ be a transition. The transition instance $\langle o, t\rangle$ is enabled in $g c$, denoted by enabled $(g c,\langle o, t\rangle)$, if

- the state configuration is stable: stable $(s c)$,

- the source state is active: $s \in A$,

- $\operatorname{Input} Q(g c, o)=\left\langle\operatorname{sig}\left[v_{1}, \ldots, v_{k}\right], \ldots\right\rangle$,

- $\operatorname{eval}\left(g c^{\star}, o, g\right)=$ true, where $g c^{\star}$ is equal to $g c$ except that in $o$, the message $\operatorname{sig}\left[v_{1}, \ldots, v_{k}\right]$ has been received: $\operatorname{Input} Q\left(g c^{\star}, o\right)=$ dequeue $(\operatorname{Input} Q(g c, o))$ and $\forall 1 \leq i \leq k: \operatorname{AttrVal}\left(g c^{\star}, o, x_{i}\right)=$ $v_{i}$ (the formal parameters in the signal trigger are required to be attributes in the class of $o$ ),

- no prioritized transition is enabled [22, p. 608], i.e. there is no enabled transition deeper in the hierarchy: $\nexists t^{\prime} \in \mathcal{T}:$ source $\left(t^{\prime}\right) \in$ descendants $(s) \cap A \wedge \operatorname{enabled}\left(g c,\left\langle o, t^{\prime}\right\rangle\right)$, and

- the message is not being deferred by a state at a deeper level: $\nexists s^{\prime \prime} \in \operatorname{descendants}(s) \cap\left(\mathcal{S}_{s i} \cup \mathcal{S}_{c o}\right) \cap A: \operatorname{sig} \in \operatorname{defers}\left(s^{\prime \prime}\right)$.

If enabled $(g c,\langle o, t\rangle)$ holds, then $\left\langle g c,\langle o, t\rangle, g c^{\prime}\right\rangle \in \Delta$, where the global configuration $g c^{\prime}$ is equal to $g c^{\prime \prime}=\operatorname{exec}\left(g c^{\star}, o, e\right)$ except that the state configuration is updated: StateConf $\left(g c^{\prime}, o\right)=\operatorname{succ-conf}(s c, t)$, and the deferred queue is flushed to the input queue: $\operatorname{Defer} Q\left(g c^{\prime}, o\right)=\langle\rangle$ and $\operatorname{Input} Q\left(g c^{\prime}, o\right)=\operatorname{concat}\left(\operatorname{Defer} Q(g c, o), \operatorname{Input} Q\left(g c^{\prime \prime}, o\right)\right)$.

- Deferring. If no transition instance is enabled, then the first message in the input queue can be deferred. Formally, the deferring instance $\langle o$, DEFER $\rangle$ is enabled in $g c$, denoted by enabled $(g c,\langle o$, DEFER $\rangle)$, if

- the state configuration is stable: stable $(s c)$,

- $\operatorname{Input} Q(g c, o)=\left\langle\operatorname{sig}\left[v_{1}, \ldots, v_{k}\right], \ldots\right\rangle$,

- $\nexists t \in \mathcal{T}: \operatorname{enabled}(g c,\langle o, t\rangle)$, and

- there is an active state deferring the message: $\exists s \in\left(\mathcal{S}_{s i} \cup \mathcal{S}_{c o}\right) \cap A: \operatorname{sig} \in \operatorname{defers}(s)$. 
If enabled $(g c,\langle o$, DEFER $\rangle)$ holds, then $\left\langle g c,\langle o\right.$, DEFER $\left.\rangle, g c^{\prime}\right\rangle \in \Delta$, where $g c^{\prime}$ is equal to $g c$ except that Input $Q\left(g c^{\prime}, o\right)=\operatorname{dequeue}(\operatorname{Input} Q(g c, o))$ and $\operatorname{Defer} Q\left(g c^{\prime}, o\right)=\operatorname{append}\left(\operatorname{Defer} Q(g c, o), \operatorname{sig}\left[v_{1}, \ldots, v_{k}\right]\right)$.

- Implicit consumption. If the first message in the input queue is not consumed by a transition or deferred, it can be implicitly consumed. Formally, enabled $(g c,\langle o$, IMPCONS $\rangle)$ holds if

- the state configuration is stable: stable $(s c)$,

- $\operatorname{Input} Q(g c, o)=\left\langle\operatorname{sig}\left[v_{1}, \ldots, v_{k}\right], \ldots\right\rangle$,

- $\nexists t \in \mathcal{T}: \operatorname{enabled}(g c,\langle o, t\rangle)$, and

- $\operatorname{enabled}(g c,\langle o$, DEFER $\rangle)$ does not hold.

If enabled ( $g c,\langle o$, IMPCONS $\rangle)$ holds, then $\left\langle g c,\langle o\right.$, IMPCONS $\left.\rangle, g c^{\prime}\right\rangle \in \Delta$, where $g c^{\prime}$ is equal to $g c$ except that the first message has been consumed: $\operatorname{Input} Q\left(g c^{\prime}, o\right)=\operatorname{dequeue}(\operatorname{Input} Q(g c, o))$.

- Completion transitions. Implicit completion events are consumed until a stable state configuration is reached. Formally, for each transition $t=\left\langle s, \sigma, g, e, s^{\prime}\right\rangle \in \mathcal{T}$ such that $\sigma=\tau$, the completion transition instance $\langle o, t\rangle$ is enabled in $g c$, denoted by enabled $(g c,\langle o, t\rangle)$, if

- the source is active: $s \in A$,

- either (i) the source is a pseudostate: $s \in \mathcal{S}_{\text {in }} \cup \mathcal{S}_{c h}$ or (ii) the state configuration is in RTC step but not in compound transition and the source state $s$ ready to consume its completion event: $\neg \operatorname{inct}(s c) \wedge \operatorname{inrtc}(s c) \wedge s \in \mathcal{S}_{s i} \cup \mathcal{S}_{c o} \wedge$ ceready $(s c, s)$, and

- the guard condition holds: $\operatorname{eval}(g c, o, g)=$ true.

If enabled $(g c,\langle o, t\rangle)$ holds, then $\left\langle g c,\langle o, t\rangle, g c^{\prime}\right\rangle \in \Delta$, where $g c^{\prime}$ is equal to $\operatorname{exec}(g c, o, e)$ except that the state configuration is updated: StateConf $\left(g c^{\prime}, o\right)=\operatorname{succ-conf}(s c, t)$.

- Quiescing. If a completion sensitive state $s \in \mathcal{S}_{C S}$ is ready to consume its completion event but no outgoing completion transition is enabled, the state can quiesce (i.e. implicitly consume the completion event). Formally, enabled $\left(g c,\left\langle o\right.\right.$, QUIESCE$\left.\left._{s}\right\rangle\right)$ holds if

- the state configuration is in RTC but not in compound transition: $\operatorname{inrtc}(s c) \wedge \neg \operatorname{inct}(s c)$,

- the state is active and ready to consume its completion event: $s \in \mathcal{S}_{C S} \cap A \wedge$ ceready $(s c, s)$, and

- $\nexists t \in \mathcal{T}: \operatorname{source}(t)=s \wedge$ triggersig $(t)=\tau \wedge \operatorname{enabled}(g c,\langle o, t\rangle)$.

If enabled $\left(g c,\left\langle o\right.\right.$, QUIESCE $\left.\left._{s}\right\rangle\right)$ holds, then $\left\langle g c,\left\langle o\right.\right.$, QUIESCE $\left.\left._{s}\right\rangle, g c^{\prime}\right\rangle \in$ $\Delta$, where $g c^{\prime}$ is equal to $g c$ except that state $s$ has become quiescent: StateConf $\left(g c^{\prime}, o\right)=\langle A, Q \cup\{s\}\rangle$. 


\section{ENCODING SYSTEM BEHAVIOR}

The symbolic encoding of the state space transition relation is based on constraints involving state variables, whose valuation represents a global configuration, next-state variables, whose valuation represents the global configuration after executing one step, input variables used to capture non-determinism and whose values are only limited by the constraints, and auxiliary derived functions defined over the variables. All variables and functions have Boolean values unless otherwise stated. To keep the state space finite, we assume that $\operatorname{dom}(T)$ is finite for each type $T$ and restrict the analysis to bounded global configurations, where for each object, the total number of messages in its input and deferred queues is limited by the constant QSIZE. This implies that all non-Boolean variables have finite domains and thus can be booleanized to enable the use of SAT- and BDD-based techniques.

More specifically, we define an encoding such that, given a valuation for state variables that represents a bounded global configuration $g c$ and an arbitrary valuation for next-state variables, there exists a valuation for input variables that satisfies all the constraints if and only if the valuation for next-state variables represents a bounded global configuration $g c^{\prime}$ such that $\left\langle g c, a, g c^{\prime}\right\rangle \in \Delta$ for some annotation $a$.

\subsection{State Variables}

Let $g c$ be a bounded global configuration. Let the state configuration of an object $o \in O$ be $\langle A, Q\rangle=\operatorname{State} \operatorname{Conf}(g c, o)$, and let the numbers $d$ and $n$ and messages $M_{0}, \ldots, M_{n-1}$ be such that the deferred queue of $o$ is $\left\langle M_{0}, \ldots, M_{d-1}\right\rangle=\operatorname{Defer} Q(g c, o)$ and the input queue is $\left\langle M_{d}, \ldots, M_{n-1}\right\rangle=$ Input $Q(g c, o)$. Because $g c$ is bounded, we have $0 \leq d \leq n \leq$ QSIZE. For each object, the set of state variables contains five kinds of elements, with values derived from $g c$ as follows.

- Active $(o, s)$, where $s$ is a state vertex in the state machine of $o$, is true if and only if $s$ is active, i.e. $s \in A$.

- Quiescent $(o, s)$, where $s$ is a completion sensitive state in the state machine of $o$, is true if and only if $s \in Q$.

- $\operatorname{AttrVal}(o, x)$, where $x \in \operatorname{Attrs}(\operatorname{Class}(o))$, has domain $\operatorname{dom}(x)$ and value $\operatorname{Attr} \operatorname{Val}(g c, o, x)$. We omit all details of data handling and do not reference these variables in the encoding.

- Queue $(o, k)$, where $0 \leq k<$ QSIZE, has domain Msgs $\cup$ none. Its value is $M_{k}$ if $k<n$, and none otherwise. In other words, the sequence $\langle$ Queue $(o, 0), \ldots$, Queue $(o$, QSIZE -1$)\rangle$ consists of the deferred queue, followed by the input queue, followed by zero or more none entries.

- $\operatorname{QPos}(o)$ has domain $\{0,1, \ldots$, QSIZE $\}$ and value $d$.

The corresponding next-state variables are denoted by next $(\operatorname{Active}(o, s))$, next (Quiescent $(o, s)$ ), etc. 


\subsection{Queues and Messages}

Let $o \in O$ be an object. We restrict the model so that one transition can send at most one new message to each object (this can be circumvented by splitting non-complying transitions to segments and adding choice pseudostates between them). The function QNewMsg(o) with values in Msgs $\cup$ \{none\} evaluates to the message being sent to $o$ during the step $\left\langle g c, a, g c^{\prime}\right\rangle$, or none if no message is being sent. The definition of QNewMsg $(o)$ depends on the action language statements, which are not considered here.

The function CurrentMsg $(o)$ with domain Msgs $\cup$ \{none\} contains the first message in the input queue, or none if the input queue is empty:

$$
\text { CurrentMsg }(o):=\text { if } \begin{cases}\text { QPos }(o)=0 & \text { : Queue }(o, 0) \\ \vdots & \vdots \\ \text { QPos }(o)=\text { QSIZE }-1 & : \text { Queue }(o, \text { QSIZE }-1) \\ \text { else } & \text { : none. }\end{cases}
$$

The input variable Dispatch $(o)$ with domain Sigs $\cup\{$ none $\}$ determines the signal in the message (if any) being consumed by $o$. The signal must be present in the first message in the input queue, so we add for each sig $\in$ Sigs the constraint

$$
(\operatorname{Dispatch}(o)=\operatorname{sig}) \Rightarrow(\text { CurrentMsg }(o)=\operatorname{sig}[\ldots]) .
$$

The queues interface with other parts via the following operations: (i) removing the first message from the input queue and discarding it, triggered by the predicate $Q \operatorname{Rem}(o)$, (ii) moving the first message from the input queue to the deferred queue, triggered by $Q \operatorname{Defer}(o)$, (iii) flushing the entire contents of the deferred queue to the input queue, triggered by QFlush $(o)$, and (iv) adding the new message QNewMsg(o) to the input queue. Any combination of operations is allowed to occur in the same step as long as their order respects the list above, and cases (i) and (ii) do not occur in the same step. The functions QRem $(o)$, QDefer $(o)$, and QFlush $(o)$ are defined in Sect. 3.3.

Operation (iii) above corresponds to resetting QPos $(o)$ to zero, and operation (ii) corresponds to incrementing $\mathrm{QPos}(o)$, formalized by the constraint

$$
\operatorname{next}(\mathrm{QPos}(o))=\text { if } \begin{cases}\mathrm{QFlush}(o) & : 0 \\ \mathrm{QDefer}(o) & : \mathrm{QPos}(o)+1 \\ \text { else } & : \operatorname{QPos}(o) .\end{cases}
$$

Operation (i) removes the element at index QPos $(o)$ from the queue, shifting the elements at QPos $(o)+1, \ldots$, QSIZE -1 one position to the left. Operation (iv) adds the new message to the first free position, i.e. the position $k$ such that Queue $(o, k-1) \neq$ none and Queue $(o, k)=$ none. However, if operation (i) has been performed in the same step, the position must be decremented by 1 . The new queue contents for $0 \leq k<$ QSIZE is thus determined by

$$
\operatorname{next}(\text { Queue }(o, k))=\text { if } \begin{cases}N_{o}(k) & : \text { QNewMsg }(o) \\ \operatorname{QRem}(o) \wedge \operatorname{QPos}(o) \leq k & : \text { Queue }(o, k+1) \\ \text { else } & : \text { Queue }(o, k),\end{cases}
$$


where

$$
\begin{aligned}
N_{o}(k):=(\mathrm{QRem}(o) \Leftrightarrow(\text { Queue }(o, k) \neq \text { none })) & \wedge \\
(\text { Queue }(o, k-1) \neq \text { none }) & \wedge(\text { Queue }(o, k+1)=\text { none }) .
\end{aligned}
$$

The boundaries are defined as we substitute true for both Queue $(o$, QSIZE $)=$ none and Queue $(o,-1) \neq$ none. To forbid transitions to global configurations that are not bounded, we prevent the queue from overflowing by setting the constraint

$$
(\mathrm{QNewMsg}(o) \neq \text { none }) \Rightarrow(\text { Queue }(o, \operatorname{QSIZE}-1)=\text { none }) \vee Q \operatorname{Rem}(o)
$$

\subsection{Control Logic of State Machines}

Next we describe a compact symbolic encoding for the semantics of UML state machines as described in Sect. 2.3. Let $o \in O$ be an object with the state machine $\langle\mathcal{S}, \mathcal{R}$, top, container, $\mathcal{T}$, defers $\rangle$.

State Configuration Classification. As defined in Sect. 2.2, a completion sensitive state $s \in \mathcal{S}_{C S}$ is ready to consume its completion event if it is active, not quiescent, and all its regions (if any) are in final states:

$$
\begin{aligned}
& \operatorname{CEReady}(o, s):=\operatorname{Active}(o, s) \wedge \neg \text { Quiescent }(o, s) \wedge \\
& \qquad \bigwedge\{F(o, r) \mid r \in \operatorname{children}(s)\},
\end{aligned}
$$

where $F(o, r):=\bigvee\left\{\operatorname{Active}\left(o, s^{\prime}\right) \mid s^{\prime} \in \operatorname{children}(r) \cap \mathcal{S}_{f i}\right\}$ is true iff the active child state of the region $r$ is a final state.

Based on this, it is easy to define the predicates $\operatorname{InCT}(o)$ and $\operatorname{InRTC}(o)$ telling whether the state configuration is in compound transition or in RTC step, respectively:

$$
\begin{aligned}
\operatorname{InCT}(o) & :=\bigvee\left\{\operatorname{Active}(o, s) \mid s \in \mathcal{S}_{i n} \cup \mathcal{S}_{c h}\right\} \text {, and } \\
\operatorname{InRTC}(o) & :=\operatorname{InCT}(o) \bigvee \bigvee\left\{C \operatorname{CEReady}(o, s) \mid s \in \mathcal{S}_{C S}\right\} .
\end{aligned}
$$

Because an object can consume events from the input queue only if its state configuration is stable, we add the constraint

$$
\operatorname{InRTC}(o) \Rightarrow(\operatorname{Dispatch}(o)=\text { none }) .
$$

Enabledness Conditions for Transitions. We associate with each transition $t \in \mathcal{T}$ of the state machine an input variable Fire $(o, t)$ that determines whether $t$ is being fired in $o$. According to the semantics, firing a transition $t$ requires that (i) the source state is active, and (ii) the guard evaluates to true, captured by the constraint

$$
\text { Fire }(o, t) \Rightarrow \operatorname{Active}(o, \operatorname{source}(t)) \wedge \operatorname{EvalGuard}(o, t) .
$$

We assume that EvalGuard $(o, t)$ encodes the function $\operatorname{eval}\left(g c^{\star}, o, \operatorname{guard}(t)\right)$ (or $\operatorname{eval}(g c, o, \operatorname{guard}(t))$ if $t$ is a completion transition) in Sect. 2.3, and omit its formula. 
If $t$ is a completion transition whose source is a simple or composite state, it is also required that (i) the state configuration is not in a compound transition and (ii) the source state is ready to consume its completion event:

$$
\text { Fire }(o, t) \Rightarrow \neg \operatorname{InCT}(o) \wedge \text { CEReady }(o, \operatorname{source}(t)) \text {. }
$$

If $t$ is not a completion transition, then we have to ensure that there is no enabled prioritized transition and no active descendant state can defer the message. For this purpose we define an auxiliary function

$$
\begin{aligned}
& \text { Feasible }(o, s, \operatorname{sig}):=\operatorname{Active}(o, s) \wedge \\
& \neg \text { DescFeasible }(o, s, \operatorname{sig}) \wedge \neg \text { DescDeferring }(o, s, \text { sig }) \wedge \\
& \bigvee\{\text { EvalGuard }(o, t) \mid t \in R(s, \operatorname{sig})\}
\end{aligned}
$$

with $R(s$, sig $)=\{t \in \mathcal{T} \mid$ source $(t)=s \wedge \operatorname{triggersig}(t)=\operatorname{sig}\}$. Feasible $(o, s$, sig) evaluates to true iff (i) there is at least one sig-triggered transition with source $s$ and guard satisfied, and (ii) there is neither an active descendant of $s$ that can defer sig nor a prioritized enabled sig-triggered transition. The function DescFeasible $(o, v, s i g)$ is defined simply by

$$
\begin{aligned}
\operatorname{DescFeasible}(o, v, \operatorname{sig}):= & \bigvee\left\{\text { Feasible }\left(o, v^{\prime}, \operatorname{sig}\right) \vee\right. \\
& \text { DescFeasible } \left.\left(o, v^{\prime}, \operatorname{sig}\right) \mid v^{\prime} \in \operatorname{children}(v)\right\},
\end{aligned}
$$

with Feasible $(o, v, \operatorname{sig}):=$ false for $v \in \mathcal{S}_{i n} \cup \mathcal{S}_{c h} \cup \mathcal{S}_{f i} \cup \mathcal{R}$. The function DescDeferring $(o, s, s i g)$ is defined later. Now we only have to constrain that if a message with signal sig is to be consumed from the input queue and there are enabled sig-triggered transitions leaving from an active state $s \in \mathcal{S}_{s i} \cup \mathcal{S}_{c o}$, then one of them is fired:

$$
\begin{aligned}
& \bigvee\left\{\text { Fire }(o, t) \mid t \in R_{o}(s, \operatorname{sig})\right\} \Leftrightarrow \\
& (\operatorname{Dispatch}(o)=\operatorname{sig}) \wedge \text { Feasible }(o, s, \text { sig }) .
\end{aligned}
$$

Constraint (20) below ensures that at most one such transition is fired. Due to (9), a signal triggered transition can only be fired in a stable state configuration.

State Configuration Change due to Transition Firing. Perhaps the most complicated part in the symbolic encoding is the computation of successor state configurations as defined in Sect. 2.2. A state vertex $s \in \mathcal{S}$ becomes active in the next configuration if it is entered by firing a transition, and it remains active if it is not exited by a transition:

$$
n \operatorname{ext}(\operatorname{Active}(o, s)) \Leftrightarrow \operatorname{Enter}(o, s) \vee(\operatorname{Active}(o, s) \wedge \neg \operatorname{Exit}(o, s)) \text {. }
$$

A non-initial state vertex is entered if it is the target of a transition being fired, or if it is a composite state whose region is being broken in by a transition. We say that a fired transition $t$ breaks in a region $r$ if the transition cuts in through the boundary of the region in the diagram, or formally, if $r \in$ descendants (container $(t))$ and target $(t) \in \operatorname{descendants}(r)$. In Fig. 1, the 
only such transition is $t_{10}$, which breaks in region $r_{3}$. For each vertex $s \in$ $\mathcal{S} \backslash \mathcal{S}_{\text {in }}$

$$
\begin{aligned}
\operatorname{Enter}(o, s):= & \bigvee\{\operatorname{Fire}(o, t) \mid t \in \mathcal{T} \wedge \operatorname{target}(t)=s\} \vee \\
& \bigvee\{\operatorname{BreakIn}(o, r) \mid r \in \operatorname{children}(s)\},
\end{aligned}
$$

and for each region $r \in \mathcal{R}$,

$$
\begin{aligned}
\operatorname{BreakIn}(o, r):= & \bigvee\left\{\operatorname{Enter}(o, s) \mid s \in \operatorname{children}(r) \backslash \mathcal{S}_{i n}\right\} \wedge \\
& \neg \bigvee\{\operatorname{Fire}(o, t) \mid t \in \mathcal{T} \wedge \text { container }(t)=r\} .
\end{aligned}
$$

An initial pseudostate $s_{i n} \in \mathcal{S}_{\text {in }}$ is entered if its containing state is entered but its containing region is not broken in:

$$
\begin{aligned}
\operatorname{Enter}\left(o, s_{i n}\right):= & \operatorname{Enter}\left(o, \text { container }{ }^{2}\left(s_{\text {in }}\right)\right) \wedge \\
& \neg \text { BreakIn }\left(o, \text { container }\left(s_{\text {in }}\right)\right) .
\end{aligned}
$$

As a special case, Enter $\left(o, s_{i n}\right):=$ false if $s_{\text {in }} \in \mathcal{S}_{\text {in }} \cap$ children (top). For each $s \in \mathcal{S}$, the value of Exit $(o, s)$ is true iff $s$ or one of its ancestors is being exited by a transition, defined by

$$
\operatorname{Exit}(o, s):=\operatorname{Exit}\left(o, \text { container }^{2}(s)\right) \vee \bigvee X_{o}(s),
$$

where $X_{o}(s)$ is the set consisting of each Fire $(o, t)$ such that $t \in \mathcal{T}$ and $s \in$

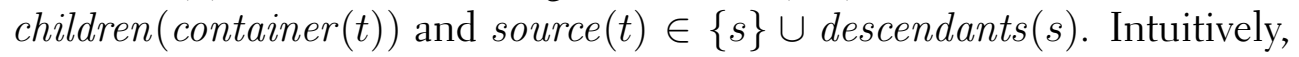
Fire $(o, t)$ is in the set $X_{o}(s)$ iff $s$ is the outermost state vertex exited when $t$ is fired. For example in Fig. $1, X_{o}\left(A_{2}\right)=\left\{\right.$ Fire $\left(o, t_{10}\right)$, Fire $\left.\left(o, t_{11}\right)\right\}$ and $X_{o}\left(B_{2}\right)=\left\{\right.$ Fire $\left.\left(o, t_{8}\right)\right\}$.

To avoid firing several transitions from the same state at the same time, for each $s \in \mathcal{S}$ the constraint

$$
\text { AtMostOne }\left(\left\{\text { Exit }\left(o, \text { container }^{2}(s)\right)\right\} \cup X_{o}(s)\right)
$$

is added that allows at most one of the disjuncts in (19) to be true. A predicate of the form AtMostOne $(P)$ evaluates to true if and only if zero or one of the predicates in set $P$ evaluates to true. This can be expressed with $\mathcal{O}(|P|)$ binary Boolean connectives. In (19) and (20), the term Exit (o, container $\left.{ }^{2}(s)\right)$ is omitted if container $(s)=$ top.

Quiescing. We associate with each completion sensitive state $s \in \mathcal{S}_{C S}$ an input variable Quiesce $(o, s)$ that indicates whether $s$ is becoming quiescent. The state $s$ becomes quiescent when Quiesce $(o, s)$ is true, and it remains quiescent until it is exited:

$$
\text { next (Quiescent }(o, s)) \Leftrightarrow \neg \operatorname{Exit}(o, s) \wedge(\text { Quiesce }(o, s) \vee \text { Quiescent }(o, s)) \text {. }
$$

The following constraint ensures that quiescing can only happen when (i) there are no active pseudostates and (ii) the state is ready to consume its completion event but none of the outgoing completion transitions is enabled:

$$
\begin{aligned}
\text { Quiesce }(o, s) \Rightarrow & \neg \operatorname{InCT}(o) \wedge \operatorname{CEReady}(o, s) \wedge \\
& \neg \bigvee\left\{\text { EvalGuard }(o, t) \mid t \in R_{\tau}(s)\right\}
\end{aligned}
$$


with $R_{\tau}(s)=\{t \in \mathcal{T} \mid$ source $(t)=s \wedge$ triggersig $(t)=\tau\}$. Together with (8) this ensures that quiescing can only happen in an RTC step: Quiesce $(o, s)$ implies InRTC $(o)$.

Deferring. For each signal sig $\in$ Sigs and each simple or composite state $s \in \mathcal{S}_{s i} \cup \mathcal{S}_{c o}$, the function

$$
\text { Deferring }(o, s, \operatorname{sig}):= \begin{cases}\operatorname{Active}(o, s) & \text { if } \operatorname{sig} \in \operatorname{defers}(s), \\ \text { false } & \text { otherwise }\end{cases}
$$

is true iff $s$ is active and can defer the signal sig. Define Deferring $(o, v, \operatorname{sig}):=$ false if $v \in \mathcal{S}_{i n} \cup \mathcal{S}_{c h} \cup \mathcal{S}_{f i} \cup \mathcal{R}$. Now for each $v \in \mathcal{S} \cup \mathcal{R}$,

$$
\begin{aligned}
\text { Desc Deferring }(o, v, \operatorname{sig}):= & \bigvee\left\{\text { Deferring }\left(o, v^{\prime}, \operatorname{sig}\right) \vee\right. \\
& \text { DescDeferring } \left.\left(o, v^{\prime}, \operatorname{sig}\right) \mid v^{\prime} \in \operatorname{children}(v)\right\}
\end{aligned}
$$

is true iff $v$ has an active descendant that can defer sig.

The object $o$ defers the first message in its input queue if the message is dispatched, there is an active deferring state, and no transition is consuming the message:

$$
\begin{aligned}
\operatorname{Defer}(o, \operatorname{sig}):= & \operatorname{DescDeferring}(o, \text { top }, \text { sig }) \wedge \\
& \neg \operatorname{DescFeasible}(o, \text { top }, \operatorname{sig}) \wedge(\operatorname{Dispatch}(o)=\operatorname{sig}), \\
\operatorname{QDefer}(o):= & \bigvee\{\operatorname{Defer}(o, \text { sig }) \mid \operatorname{sig} \in \operatorname{Sigs}\} .
\end{aligned}
$$

Note that QDefer $(o)$ implies Dispatch $(o) \neq$ none and thus (9) ensures that deferring can only happen in a stable state configuration, i.e. QDefer $(o)$ implies $\neg \operatorname{InRTC}(o)$.

Putting it All Together. The constraints so far do not prevent simultaneous firing of completion transitions or quiescing of states. This is fixed by the constraint

$$
\text { AtMostOne }\left(C_{o} \cup Q_{o}\right) \text {, }
$$

where $C_{o}=\{$ Fire $(o, t) \mid t \in \mathcal{T} \wedge$ triggersig $(t)=\tau\}$ corresponds to $o$ firing a completion transition and $Q_{o}=\left\{\right.$ Quiesce $\left.(o, s) \mid s \in \mathcal{S}_{C S}\right\}$ corresponds to $o$ quiescing a state.

When examining the system as a whole, an object is scheduled if it is consuming a message, firing a completion transition, or quiescing a state. The object is ready for execution if one of these occurrences is enabled. The system is in a deadlock if no object is ready. These are formalized below.

$$
\begin{aligned}
\operatorname{Scheduled}(o) & :=(\operatorname{Dispatch}(o) \neq \text { none }) \vee \bigvee\left(C_{o} \cup Q_{o}\right), \\
\operatorname{Ready}(o) & :=(\text { CurrentMsg }(o) \neq \text { none }) \vee \operatorname{InRTC}(o), \\
\operatorname{Deadlock}() & :=\neg \bigvee\{\operatorname{Ready}(o) \mid o \in O\} .
\end{aligned}
$$


The functions needed by input queue encoding, presented in the previous section, are defined as follows:

$$
\begin{aligned}
\operatorname{QFlush}(o):=\bigvee\{\text { DescFeasible }(o, \text { top }, \text { sig }) \wedge \\
(\operatorname{Dispatch}(o)=\operatorname{sig}) \mid \operatorname{sig} \in \operatorname{Sigs}\}, \\
\mathrm{QRem}(o):=(\operatorname{Dispatch}(o) \neq \text { none }) \wedge \neg Q \operatorname{Defer}(o) .
\end{aligned}
$$

To obtain an interleaving execution semantics, we must constrain that exactly one object is scheduled at a time:

$$
\begin{gathered}
\bigvee\{\operatorname{Scheduled}(o) \mid o \in O\} \text {, and } \\
\operatorname{AtMostOne}(\{\operatorname{Scheduled}(o) \mid o \in O\}) .
\end{gathered}
$$

Using this transition relation encoding, we can now use symbolic model checking tools such as NuSMV [6] to check properties of UML systems. For example, to check that a deadlock cannot be reached, we use a model checker to check that $\neg$ Deadlock() is an invariant in all executions.

\subsection{Analysis}

Assuming that the state variables represent a bounded global configuration and all constraints are satisfied, there is by (26) and (27) a unique object $o$ such that Scheduled $(o)$ holds. There are five mutually exclusive cases: (i) QFlush $(o)$ is true, consequently QRem $(o)$ is true, there is a unique $t \in \mathcal{T}$ such that Fire $(o, t)$ is true, and triggersig $(t)=\operatorname{Dispatch}(o) \neq$ none. This corresponds to the step $\left\langle g c,\langle o, t\rangle, g c^{\prime}\right\rangle \in \Delta$. (ii) $Q \operatorname{Defer}(o)$ is true, corresponding to $\left\langle g c,\langle o\right.$, DEFER $\left.\rangle, g c^{\prime}\right\rangle \in \Delta$. (iii) QFlush $(o)$ and QDefer $(o)$ are both false and $\operatorname{Dispatch}(o) \neq$ none, which corresponds to implicit consumption $\left\langle g c,\langle o\right.$, IMPCONS $\left.\rangle, g c^{\prime}\right\rangle \in \Delta$. (iv) $\operatorname{Dispatch}(o)=$ none and Fire $(o, t)$ is true for some $t$, in which case triggersig $(t)=\tau$, corresponding to $\left\langle g c,\langle o, t\rangle, g c^{\prime}\right\rangle \in$ $\Delta$. (v) $\operatorname{Dispatch}(o)=$ none and Quiesce $(o, s)$ is true for a state $s \in \mathcal{S}_{C S}$, corresponding to the quiescing step $\left\langle g c,\left\langle o\right.\right.$, QUIESCE $\left.\left._{s}\right\rangle, g c^{\prime}\right\rangle \in \Delta$.

The size of the encoding is linear in the size $|\mathcal{M}|$ of the input model, which includes the definition of all signals, state vertices, transitions, and deferrable signals. We assume that all trivial definitions have been eliminated, i.e. those derived functions that are vacuously true or false or equal to another function or variable, and constraints that are vacuously true. The queue encoding of Sect. 3.2 for a single object has size $\mathcal{O}(\mathrm{QSIZE} \cdot W)$, where $W$ is the maximum bit width of a message. Consider the control logic encoding of Sect. 3.3 for an object $o$. The definitions (16), (17), and (19) are designed so that each variable Fire $(o, t)$ appears in exactly one definition of each kind, so these are $\mathcal{O}(|\mathcal{S}|+|\mathcal{T}|)$ in size. The total size of nontrivial definitions of the form (12) and (13), summed over all $s \in \mathcal{S}$ and sig $\in$ Sigs, is $\mathcal{O}(|\mathcal{T}|)$. The total size of $(23)$ and $(24)$ is $\mathcal{O}\left(\Sigma_{s \in \mathcal{S}}|\operatorname{defers}(s)|\right)$. Other definitions are $\mathcal{O}(|\mathcal{S}|+|\mathcal{T}|+\mid$ Sigs $\mid)$ in size. Summing up, the total size of the encoding of a model without data is $\mathcal{O}(|O|($ QSIZE $\cdot W+|\mathcal{M}|))$. 
Table 1: Symbolic model checking of a hierarchical vs. flattened TV model.

\begin{tabular}{|r|c|c|c|}
\hline & $\begin{array}{c}\text { BMC ZChaff } \\
\text { time }\end{array}$ & $\begin{array}{c}\text { BMC MiniSat } \\
\text { time }\end{array}$ & $\begin{array}{c}\text { BDD Invar } \\
\text { time }\end{array}$ \\
\hline original & $16.49-54.60$ & $29.29-90.00$ & $0.63-0.67$ \\
flattened & $30.12-77.94$ & $25.16-300.63$ & $1.06-1.09$ \\
\hline
\end{tabular}

\subsection{Preliminary Evaluation}

We have implemented the symbolic encoding described above. The implementation $^{1}$ reads a UML model, stored in the XMI file format supported by the open-source meta-modeling tool Coral [1], and translates it to the input language of the NuSMV symbolic model checking tool [6]. For the experiments here we use NuSMV version 2.4.3.

To evaluate whether allowing hierarchy in the encoding is helpful, we use a variant of the TV model in [18] that is one of the standard example systems in UML model checking literature. We try to measure only the hierarchy aspect by model checking a deadlock-freedom property of a modified model in which the property does not hold-the TV stops working after it has been switched off 50 times. The symbolic encoding is evaluated on both the original hierarchical model and the corresponding flattened model; the original state machine has 12 state vertices and 13 transitions while the flattened state machine has 11 state vertices and 25 transitions. The results in Table 1 show the minimum and maximum CPU times of ten runs of (i) a state-of-the-art incremental BMC algorithm $[15,5]$ when ZChaff (version 64bit.2007.3.12) [19] and MiniSat2 (version 061208) [12] are applied as the SAT solver, and (ii) the basic BDD-based invariant checking algorithm. For this model it is clearly beneficial not to flatten the hierarchy as a preprocessing step. Our hypothesis for this behavior is that because flattening increases the number of transitions in the model, it also increases the search space of the SAT solver.

Furthermore, the experiments in [11] compare explicit-state model checking using Spin to BMC using the queue and control logic encoding presented here. The results indicate that symbolic model checking can complement explicit-state model checking even when analyzing asynchronous protocol models. Symbolic model checking also has the favorable feature that when a property violation is found, the returned counterexample execution is always short, making it simpler for the user to analyze.

Obviously, a more thorough evaluation of efficiency and bottlenecks in symbolic model checking of asynchronous message passing UML systems is needed.

\footnotetext{
${ }^{1}$ Available at http://www.tcs.hut.fi/Research/Logic/SMUML.shtml
} 


\section{CONCLUSIONS}

In this paper we have defined a semantics and a compact symbolic encoding for a class of UML models composed of asynchronously executing, message passing hierarchical state machines. This enables the use of state-of-the-art symbolic model checking techniques for the analysis of such models. Experimental results indicate that it may be beneficial to maintain the state machine hierarchy in the encoding instead of flattening the state machine before encoding.

The encoding presented in this paper uses the standard interleaving semantics, i.e. at most one object can fire at most one transition during one time step. It is also possible to extend the encoding to use so-called $\exists$ step semantics [21] so that independent transitions in several objects can be executed at the same time step; see [11] for such an encoding for nonhierarchical state machines.

There are probably many ways to optimize the state machines or the encoding to make symbolic model checking more efficient. For instance, transitions leaving the initial state of a region could in many cases be eliminated in the encoding phase. Furthermore, in order to analyze systems with more data attributes, reduction techniques such as slicing and data abstraction should also be applied.

\section{Acknowledgements}

This work has been financially supported by Tekes (Finnish Funding Agency for Technology and Innovation), Nokia, Conformiq, Mipro, and Helsinki Graduate School in Computer Science and Engineering.

\section{REFERENCES}

[1] Marcus Alanen and Ivan Porres. Coral: A metamodel kernel for transformation engines. In Proc. Second European Workshop on Model Driven Architecture (MDA), number 17-04 in Tech. Report, pages 165-170. Computing Laboratory, Univ. of Kent, Sep 2004.

[2] Michael Balser, Simon Bäumler, Alexander Knapp, Wolfgang Reif, and Andreas Thums. Interactive verification of UML state machines. In ICFEM'04, volume 3308 of LNCS, pages 343-448. Springer, 2004.

[3] Purandar Bhaduri and S. Ramesh. Model checking of statechart models: Survey and research directions. Computing Research Repository, arXiv:cs/0407038vl [cs.SE], 2004.

[4] Armin Biere, Alessandro Cimatti, Edmund M. Clarke, and Yunshan Zhu. Symbolic model checking without BDDs. In TACAS 1999, volume 1579 of LNCS, pages 193-207. Springer, 1999.

[5] Armin Biere, Keijo Heljanko, Tommi Junttila, Timo Latvala, and Viktor Schuppan. Linear encodings of bounded LTL model checking. Logical Methods in Computer Science, 2(5:5), 2006. 
[6] A. Cimatti, E. Clarke, E. Giunchiglia, F. Giunchiglia, M. Pistore, M. Roveri, R. Sebastiani, and A. Tacchella. NuSMV version 2: An opensource tool for symbolic model checking. In $C A V^{\prime} 02$, volume 2404 of LNCS, pages 359-364. Springer, 2002.

[7] Edmund Clarke, Orna Grumberg, and Doron Peled. Model Checking. The MIT Press, 1999.

[8] Michelle L. Crane and Juergen Dingel. On the semantics of UML state machines: Categorization and comparison. Technical Report 2005-501, School of Computing, Queen's University, Kingston, Ontario, Canada, 2005.

[9] Werner Damm, Bernhard Josko, Amir Pnueli, and Angelika Votintseva. A discrete-time UML semantics for concurrency and communication in safety-critical applications. Science of Computer Programming, 55:81-115, 2005.

[10] Jori Dubrovin. Jumbala - an action language for UML state machines. Research Report A101, Helsinki University of Technology, Laboratory for Theoretical Computer Science, 2006.

[11] Jori Dubrovin, Tommi Junttila, and Keijo Heljanko. Symbolic step encodings for object based communicating state machines. Technical Report B24, Helsinki University of Technology, Laboratory for Theoretical Computer Science, 2007.

[12] N. Eén and N. Sörensson. An extensible SAT-solver. In SAT'03, volume 2919 of LNCS, pages 502-518. Springer, 2004.

[13] Harald Fecher and Jens Schönborn. UML 2.0 state machines: Complete formal semantics via core state machines. In FMICS and PDMC 2006, volume 4346 of LNCS, pages 244-260. Springer, 2007.

[14] Stefania Gnesi, Diego Latella, and Mieke Massink. Modular semantics for a UML statechart diagrams kernel and its extension to multicharts and branching time model-checking. The Journal of Logic and Algebraic Programming, 51:43-75, 2002.

[15] Keijo Heljanko, Tommi Junttila, and Timo Latvala. Incremental and complete bounded model checking for full PLTL. In CAV 2005, volume 3576 of LNCS, pages 98-111. Springer, 2005.

[16] Toni Jussila, Jori Dubrovin, Tommi Junttila, Timo Latvala, and Ivan Porres. Model checking dynamic and hierarchical UML state machines. In Proc. MoDeV ${ }^{2}$ a: Model Development, Validation and Verification, pages 94-110, 2006.

[17] Kenneth L. McMillan. Symbolic Model Checking. Kluwer Academic Publishers, 1993.

[18] Erich Mikk, Yassine Lakhnech, Michael Siegel, and Gerard J. Holzmann. Implementing statecharts in Promela/Spin. In WIFT'98, pages 90-101. IEEE Computer Society, 1998. 
[19] Matthew W. Moskewicz, Conor F. Madigan, Ying Zhao, Lintao Zhang, and Sharad Malik. Chaff: Engineering an efficient SAT solver. In DAC'01, pages 530-535. ACM, 2001.

[20] E. Mota, E. Clarke, A. Groce, W. Oliveira, M. Falcão, and J. Kanda. Veriagent: an approach to integrating UML and formal verification tools. Electronic Notes in Theoretical Computer Science, 95:111-129, 2004.

[21] Jussi Rintanen, Keijo Heljanko, and Ilkka Niemelä. Planning as satisfiability: parallel plans and algorithms for plan search. Artificial Intelligence, 170(12-13):1031-1080, 2006.

[22] James Rumbaugh, Ivar Jacobson, and Grady Booch. The Unified Modeling Language Reference Manual. Addison-Wesley, second edition, 2004.

[23] Sara Van Langenhove. Towards the Correctness of Software Behavior in UML - A Model Checking Approach based on Slicing. PhD thesis, Universiteit Gent, May 2006. 


HELSINKI UNIVERSITY OF TECHNOLOGY LABORATORY FOR THEORETICAL COMPUTER SCIENCE TECHNICAL REPORTS

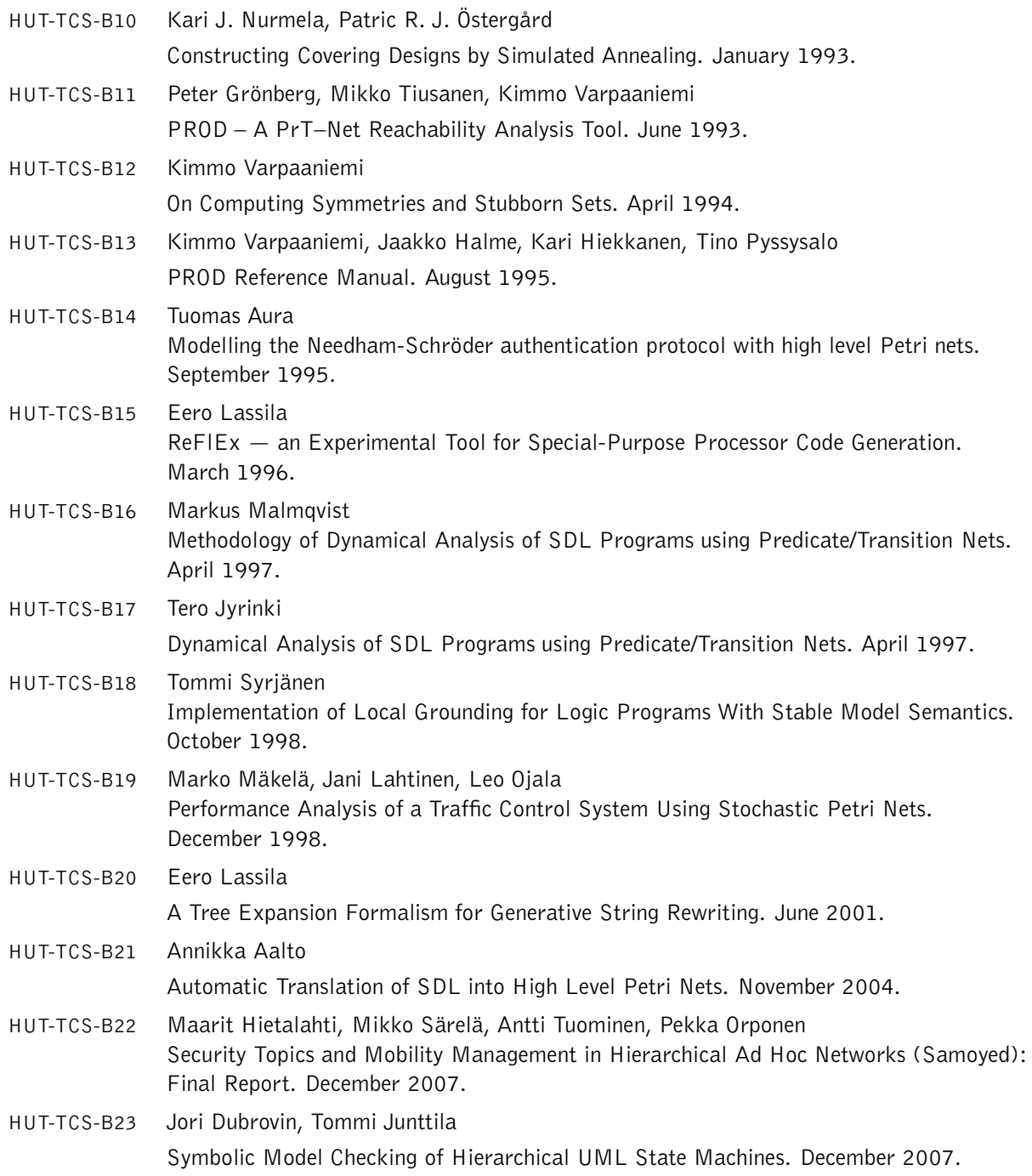

